\title{
QUATERNIONIC KAEHLER MANIFOLDS ${ }^{1}$
}

BY

\author{
LEE WHITT
}

\begin{abstract}
ABSTRACr. The topological classification of 4- and 8- (real) dimensional compact quaternionic Kaehler manifolds is given. There is only the torus in dimension 4. In dimension 8 , there are 12 homeomorphism classes; representatives are given explicitly.
\end{abstract}

In this paper, we will study compact manifolds modeled on the quaternions which admit a Kaehler metric. It is easily seen that these manifolds have zero Riemannian curvature (cf. [8]) and hence are quotients $\mathbf{R}^{4 n} / \Gamma$, where $\Gamma$ acts as a group of covering transformations preserving the Euclidean distance in $\mathbf{R}^{4 n}$. In $\$ 2$ and $\S 4$, we study $\Gamma$ to give the topological classification of compact quaternionic Kaehler manifolds in real dimensions 4 and 8 . In dimension 4 , there is only the torus. This result was obtained by Sommese [9] under conditions weaker than ours. In dimension 8 , there are 12 homeomorphism classes.

It should be noted that there are several definitions of a quaternionic Kaehler manifold. All of them, except the one considered herein, model the tangent bundle (rather than the underlying manifold) on the quaternions. For example, one can require two anticommuting complex Kaehler structures $J$ and $K$, in which case the holonomy is a subgroup of the symplectic group. According to Yau [12], these manifolds need not be Riemannian flat. Now $J$ (or $K$ ) has constant components (as a $(1,1)$ tensor) in some coordinate neighborhood, but we cannot necessarily find a neighborhood for both $J$ and $K$ simultaneously. By modeling the manifold on the quaterions, we are requiring a coordinate system so that both $J$ and $K$ have constant components.

I would like to thank the referee for bringing Auslander [0] to my attention.

\section{Preliminaries.}

1.1 Algebra. The group $E(n)$ of rigid motions of $\mathbf{R}^{n}$ is, by definition, the group of diffeomorphisms of $\mathbf{R}^{n}$ which preserves the Euclidean distance. Relative to a choice of origin $\vec{o} \in \mathbf{R}^{n}$, we can write $E(n)$ as a semidirect product $O(n) \cdot \mathbf{R}^{n}$. If $\alpha \in E(n)$, then $\alpha=(A, a)$ with $A \in O(n)$ orthogonal, $a \in \mathbf{R}^{n}$ translation by $a$, and $(A, a) r=$ $A r+a$ for $r \in \mathbf{R}^{n}$. The product rule is $(A, a)(B, b)=(A B, A b+a)$.

For any $\alpha \in E(n)$, there is a choice of origin for which $\alpha=(A, a)$ satisfies $A(a)=a$. In particular, if $\alpha$ has no fixed points, then $a \neq$ origin and $A$ has the eigenvalue +1 . To see this, if $\alpha=(A, x)$ for some choice of origin, then we may

Received by the editors June 21, 1978 and, in revised form, August 1, 1978 and April 30, 1981. 1980 Mathematics Subject Classification. Primary 53C25, 53C55.

${ }^{1}$ This work was supported in part by NSF Grant MC577-04152. 
choose $y$ so that $(A-I) x=(A-I)^{2} y$. Translating the origin to $y, \alpha$ becomes

$$
(I, y)(A, x)(I, y)^{-1}=(A, x-(A-I) y) .
$$

One easily checks that for $a=x-(A-I) y$, we have $A(a)=a$.

Let $\Gamma \subset E(n)$ be a discrete uniform subgroup without elements of finite order. It can be shown [11, p. 99] that these are the precise requirements for the action of $\Gamma$ on $\mathbf{R}^{n}$ to be properly discontinuous, have compact quotient space, and be free, respectively. Hence the quotient $M=\mathbf{R}^{n} / \Gamma$ is a compact $n$-manifold with universal covering space $\mathbf{R}^{n}$, covering transformations $\Gamma$, and fundamental group $\pi_{1}(M)=\Gamma$. Since $\Gamma$ preserves the standard (flat) metric on $\mathbf{R}^{n}$, the quotient $\mathbf{R}^{n} / \Gamma$ has an induced (flat) metric.

The converse is also true. Every compact connected flat Riemannian manifold is a quotient $M=\mathbf{R}^{n} / \Gamma$ where $\Gamma \subset E(n)$ is a discrete uniform subgroup without elements of finite order. Bieberbach [2] has shown that the free abelian normal subgroup $\Gamma^{*}=\Gamma \cap \mathbf{R}^{n}$ consisting of pure translations has finite index in $\Gamma$, i.e., the (linear) holonomy group $\Psi=\Gamma / \Gamma^{*} \subset O(n)$ is finite. It can also be shown that $\Gamma^{*}$ is maximal abelian in $\Gamma$ [4, p. 297]. If, for example, $\Gamma^{*}=\Gamma$, then $\mathbf{R}^{n} / \Gamma$ is a torus.

Auslander and Kuranishi [1] have given an algebraic characterization of $\Gamma$. An abstract group $\Gamma$ is isomorphic to the fundamental group of a compact connected flat $n$-manifold if (1) $\Gamma$ has an abelian normal subgroup $\Gamma^{*}$ which is free on $n$ generators, (2) $\Gamma^{*}$ is maximal abelian in $\Gamma$, (3) $\Gamma / \Gamma^{*}$ is finite, and (4) $\Gamma$ has no finite subgroups.

Let $\Lambda$ be the lattice of all $a \in \mathbf{R}^{n}$ for which the translation $t_{a} \in \Gamma^{*}$. Since $\Gamma^{*}$ is normal in $\Gamma, \Psi$ preserves $\Lambda$ and hence any minimal set of generators for $\Gamma^{*}$ is a vector space basis of $\mathbf{R}^{n}$ relative to which the elements of $\Psi$ all have integral entries. In particular, the matrix invariants (trace, determinant, etc.) are integral. For more details, see Wolf [11, Chapter 3].

Two compact flat Riemannian manifolds $\mathbf{R}^{n} / \Gamma$ and $\mathbf{R}^{n} / \Gamma^{\prime}$ are affinely equivalent if there is an affine transformation $\phi$ such that $\phi \Gamma \phi^{-1}=\Gamma^{\prime}$, i.e., if $\Gamma$ and $\Gamma^{\prime}$ are conjugate in the group of affine transformations of $\mathbf{R}^{n}$. Bieberbach [2] proved

THEOREM 1. $\Gamma$ and $\Gamma^{\prime}$ are isomorphic as abstract groups if and only if $\mathbf{R}^{-1} / \Gamma$ and $\mathbf{R}^{n} / \Gamma^{\prime}$ are affinely equivalent.

It follows that two compact flat Riemannian manifolds are affinely equivalent if and only if they have isomorphic fundamental groups. In particular, affine equivalence is the same as topological equivalence.

1.2 Geometry. An almost quaternionic structure on a (necessarily) $4 n$-dimensional $C^{\infty}$ manifold consists of a pair of almost complex structures satisfying $J K=-K J$. The quaternions provide the standard model of an (almost) quaternionic structure on $\mathbf{R}^{4 n}$. Let $\mathbf{R}^{4 n}$ have coordinates $\left\{\left(x_{i}, y_{i}, u_{i}, v_{i}\right) \mid i=1,2, \ldots, n\right\}$; the quaternionic structure is given by

$$
J\left(\frac{\partial}{\partial x_{i}}\right)=\frac{\partial}{\partial y_{i}}, \quad K\left(\frac{\partial}{\partial x_{i}}\right)=\frac{\partial}{\partial u_{i}},
$$




$$
\begin{array}{ll}
J\left(\frac{\partial}{\partial y_{i}}\right)=-\frac{\partial}{\partial x_{i}}, & K\left(\frac{\partial}{\partial y_{i}}\right)=\frac{\partial}{\partial v_{i}} \\
J\left(\frac{\partial}{\partial u_{i}}\right)=-\frac{\partial}{\partial v_{i}}, & K\left(\frac{\partial}{\partial u_{i}}\right)=-\frac{\partial}{\partial x_{i}} \\
J\left(\frac{\partial}{\partial v_{i}}\right)=\frac{\partial}{\partial u_{i}}, & K\left(\frac{\partial}{\partial v_{i}}\right)=-\frac{\partial}{\partial y_{i}}
\end{array}
$$

A mapping $f$ between almost quaternionic manifolds is quaternionic if the differential $d f$ commutes with the almost quaternionic structures. A quaternionic manifold is one which is covered by open sets $\left\{U_{i}\right\}$ with diffeomorphisms $\left\{\phi_{i}: U_{i} \rightarrow \mathbf{R}^{4 n}\right\}$ such that $\phi_{j} \circ \phi_{i}^{-1}: \phi_{i}\left(U_{i} \cap U_{j}\right) \rightarrow \phi_{j}\left(U_{i} \cap U_{j}\right)$ is a quaternionic mapping with respect to the above standard structure on $\mathbf{R}^{4 n}$. Clearly, a quaternionic manifold has a pair of anticommuting complex structures $J$ and $K$. A quaternionic Kaehler manifold is a quaternionic Riemannian manifold such that $J$ and $K$ preserve the metric $g$ and are parallel with respect to the Riemannian connection $\nabla$, i.e., $g(J X, J Y)=g(X, Y)$, $g(K X, K Y)=g(X, Y), \nabla_{X}(J Y)=J \nabla_{X} Y$, and $\nabla_{X}(K Y)=K \nabla_{X} Y$ for all tangent vector fields $X$ and $Y$.

LEMMA 1. A quaternionic Kaehler manifold $M$ is Riemannian flat.

Proof. Consider a quaternionic coordinate system $x_{1}, \ldots, x_{4 n}$ (so that $J$ and $K$ have constant components as $(1,1)$ tensors), and let $X, Y$ be constant coefficient linear combinations of the coordinate vector fields $\partial / \partial x_{i}$. Hence $\left[Z_{1}, Z_{2}\right]=0$ for $Z_{i}$ equal to $X, Y, J X, J Y, K X$, or $K Y, i=1,2$. Now, using the symmetry of $\nabla$, and the parallelism of $J$ and $K$, we have

$$
\begin{aligned}
0 & =-\frac{1}{2} J K([K Y, J X]-J[K Y, X]-K[Y, J X]+K J[Y, X]) \\
= & -\frac{1}{2} J K\left(J \nabla_{K Y} X-K \nabla_{J X} Y-J \nabla_{K Y} X+J K \nabla_{X} Y-K J \nabla_{Y} X\right. \\
& \left.\quad+K \nabla_{J X} Y+K J \nabla_{Y} X-K J \nabla_{X} Y\right) \\
= & -\frac{1}{2} J K\left(2 J K \nabla_{X} Y\right) \\
= & \nabla_{X} Y .
\end{aligned}
$$

Similarly $\nabla_{Y} X=\nabla_{X} X=\nabla_{Y} Y=0$, and hence

$$
g(R(X, Y) X, Y)=g\left(\nabla_{X} \nabla_{Y} X-\nabla_{Y} \nabla_{X} X-\nabla_{[X, Y]} X, Y\right)=0 \text {. Q.E.D. }
$$

Next, we will define the holonomy group $\Psi$ on an arbitrary Riemannian manifold $N$. It is a pleasant surprise that for a compact connected flat Riemannian manifold, this holonomy agrees with the holonomy in \$1.1 (see [11, Lemma 3.4.4]).

Given $p \in N$ and a $C^{\infty}$ closed curve $\sigma:[0,1] \rightarrow N, \sigma(0)=\sigma(1)=p$, we construct the parallel translation $\tau_{\sigma}: T_{p} N \rightarrow T_{p} N$ as follows. If $v \in T_{p} N$, then we may extend $v$ along $\sigma$ to $\tilde{v}$ so that $\nabla_{\sigma^{\prime}} \tilde{v}=0$, where $\sigma^{\prime}$ is the tangent field to $\sigma$ (in local coordinates, the equation $\nabla_{\sigma^{\prime}}, \tilde{v}=0$ is a system of linear differential equations which can be solved uniquely given the initial data $v$ ). We define $\tau_{\sigma}(v)=\tilde{v}_{\sigma(1)}$ where $\tilde{v}_{\sigma(0)}=v$. Since the defining equations of $\tilde{v}$ are linear, it follows that $\tau_{\sigma}$ is a linear transformation. In fact, $\tau_{\sigma}$ is an orthogonal transformation for the connection $\nabla$ is Riemannian. 
These $\tau_{\sigma}$ form the (linear) holonomy group $\Psi_{p}$. The product is composition, or equivalently addition of curves, and the inverse $\tau_{\sigma}^{-1}$ is obtained by reversing the parametrization of $\sigma$. If $q \in N$ and $\beta$ is a curve from $p$ to $q$, then $\tau \rightarrow \tau_{\beta} \tau \tau_{\beta}^{-1}$ is an isomorphism of $\Psi_{p}$ and $\Psi_{q}$. Hence, the holonomy group $\Psi$ is independent of the point.

Observe that if $J$ is an almost complex structure on a Riemannian manifold satisfying $\nabla J=0$, then $J$ commutes with $\Psi$. Hence, if $\mathbf{R}^{4 n} / \Gamma$ is a quaternionic Kaehler manifold, then $\Gamma / \Gamma^{*}$ and $\{J, K\}$ commute. In the next section, we will use this to show that for $n=1, \Psi$ is the trivial group $\{e\}$, or equivalently, $\Gamma=\Gamma^{*}$. In $\S 4$, we will show that for $n=2, \Psi$ is either $\{e\}, \mathbf{Z}_{2}, \mathbf{Z}_{3}, \mathbf{Z}_{4}$, or $\mathbf{Z}_{6}$.

2. Classification in 4 real dimensions. According to Lemma 1 , a quaternionic Kaehler manifold $M$ is Riemannian flat. If $M$ is compact and connected, then it follows that $M=\mathbf{R}^{4 n} / \Gamma$, where $\Gamma \subset E(4 n)$ is a discrete uniform subgroup without elements of finite order. To prove our classification theorems, we will first classify the holonomy $\Psi=\Gamma / \Gamma^{*}$, and then determine the (affine equivalence classes of) lattices $\Lambda$ invariant under $\Psi$. In dimension $4, \Psi$ is the trivial group and all lattices are invariant and equivalent.

THEOREM 2. If $M$ is a compact connected quaternionic Kaehler manifold of real dimension 4, then $M$ is a torus.

Proof. By the above remarks, we have $M=\mathbf{R}^{4} / \Gamma$ where $\Gamma \subset E(4)$. For $\alpha \in \Gamma$, we write $\alpha=(A, a)$ with $A(a)=a \neq 0$, and $A \in \Psi$. Choose $\{a, J a, K a, J K a\}$ as a basis for $\mathbf{R}^{4}$. Since $J$ and $K$ commute with $A \in \Psi$, it follows that $A=I$. Hence $\Psi=\{e\}, \Gamma$ consists of pure translations, and $M$ is a torus. Q.E.D.

Conversely, every 4-torus inherits the standard quaternionic Kaehler structure on $\mathbf{R}^{4}$ described in $\$ 1.2$ (since this structure is invariant under translation). We remark that Sommese [9] has shown that if $J$ is complex Kaehler and $K$ is complex (but not necessarily Kaehler), then $M$ is still a torus.

\section{Algebra.}

Proposition 1. Let $\zeta_{n}$ be a primitive nth root of unity. If the real part of $\zeta_{n}$ is rational, then $n=1,2,3,4$, or 6 .

Proof (ImProved by THE REFEREe). We adjoin $\zeta_{n}$ to the rationals $\mathbf{Q}$ and obtain the extension $\mathbf{Q}\left(\zeta_{n}\right)$. The degree of this extension is given by $\left[\mathbf{Q}\left(\zeta_{n}\right): \mathbf{Q}\right]=\phi(n)$, the Euler phi functions. If $\zeta_{n}=\alpha+i \beta$, with $\alpha$ rational, then $\zeta_{n}^{2}-2 \alpha \zeta_{n}+1=0$, and hence $\phi(n) \leqslant 2$. The phi function is given by $\phi(n)=\prod_{p \mid n} p^{r-1}(p-1)$ where $p^{r}$ divides $n$, but $p^{r+1}$ does not. For $\phi(n) \leqslant 2$, it is easy to see that $n=1,2,3,4$, or 6 . The roots are $1,-1,-\frac{1}{2} \pm \frac{\sqrt{3}}{2} i, \pm i$, or $\frac{1}{2} \pm \frac{\sqrt{3}}{2} i$, respectively. Q.E.D.

Let $f_{n}(x)$ be the $n$th cyclotomic polynomial; the roots of $f_{n}$ are precisely the primitive $n$th roots of unity and $\operatorname{deg} f_{n}=\phi(n)$. This polynomial is irreducible and has rational coefficients. If $g(x)$ is a polynomial (with rational coefficients) satisfying $g\left(\zeta_{n}\right)=0$, then $f_{n}$ divides $g$. In particular, the roots of $g$ include all $\phi(n)$ primitive $n$th roots of unity. These considerations are important in $\S 4$ and $\S 5 \mathrm{~A}$. 
4. Classifications in 8 real dimensions. In this section, we give the affine (or equivalently topological-see Theorem 1) classification of compact quaternionic Kaehler 8-manifolds $M$. Our theorem appears quite lengthy because we have included, for $M=\mathbf{R}^{8} / \Gamma$, generators and relations for $\Gamma$ as well as a representation of $\Gamma$ as a discrete uniform subgroup of the rigid motions $E(8)$.

THEOREM 3. There are 12 affine diffeomorphism classes of compact connected quaternionic Kaehler 8-manifolds. They are represented by the manifold $\mathbf{R}^{8} / \Gamma$, where $\Gamma \subset E(8)$ is one of the 12 groups given below. Here $\Lambda$ is the translation lattice with generators $\left\{a_{1}, \ldots, a_{8}\right\}, t_{k}=t_{a_{k}}$ are translations, and $\Psi=\Gamma / \Gamma^{*}$ is the holonomy. Let $i=1,2,3,4$ and $j=5,6,7,8$, and let $E$ be the span of $\left\{a_{1}, a_{2}, a_{3}, a_{4}\right\}$.

(1) $\Psi=\{e\}$ and $\Gamma$ is generated by the translations $\left\{t_{1}, \ldots, t_{8}\right\}$.

(2.1) $\Psi=\mathbf{Z}_{2}$ and $\Gamma$ is generated by $\left\{\alpha, t_{1}, \ldots, t_{8}\right\}$ where $\alpha^{2}=t_{1}, \alpha t_{i} \alpha^{-1}=t_{i}$, and $\alpha t_{j} \alpha^{-1}=t_{j}^{-1} ;\left\langle a_{i}, a_{j}\right\rangle=0$ and $\alpha=\left(A, a_{1} / 2\right)$ with $A\left(a_{i}\right)=a_{i}$ and $A\left(a_{j}\right)=-a_{j}$.

(2.2) $\Psi=\mathbf{Z}_{2}$ and $\Gamma$ is generated by $\left\{\alpha, t_{1}, \ldots, t_{8}\right\}$ where $\alpha^{2}=t_{1}, \alpha t_{i} \alpha^{-1}=t_{i}$, $\alpha t_{k} \alpha^{-1}=t_{k}^{-1}, k=5,6,7, \alpha t_{8} \alpha^{-1}=t_{1} t_{2} t_{3} t_{4} t_{8}^{-1} ;\left\langle a_{i}, a_{k}\right\rangle=0, k=5,6,7$, the projection of $a_{8}$ on $E$ is $\frac{1}{2}\left(a_{1}+a_{2}+a_{3}+a_{4}\right)$, and $\alpha=\left(A, a_{1} / 2\right)$ with $A\left(a_{i}\right)=a_{i}$, $A\left(a_{k}\right)=-a_{k}, k=5,6,7$, and $A\left(a_{8}\right)=a_{1}+a_{2}+a_{3}+a_{4}-a_{8}$.

(2.3) $\Psi=\mathbf{Z}_{2}$ and $\Gamma$ is generated by $\left\{\alpha, t_{1}, \ldots, t_{8}\right\}$ where $\alpha^{2}=t_{1}, \alpha t_{i} \alpha^{-1}=t_{i}$, $\alpha t_{5} \alpha^{-1}=t_{5}^{-1}, \quad \alpha t_{6} \alpha^{-1}=t_{6}^{-1}, \quad \alpha t_{7} \alpha^{-1}=t_{1} t_{2} t_{3} t_{4} t_{7}^{-1}, \quad \alpha t_{8} \alpha^{-1}=t_{2} t_{8}^{-1} ; \quad\left\langle a_{i}, a_{5}\right\rangle=$ $\left\langle a_{i}, a_{6}\right\rangle=0$, the projections of $a_{7}$ and $a_{8}$ on $E$ are $\frac{1}{2}\left(a_{1}+a_{2}+a_{3}+a_{4}\right)$ and $\frac{1}{2} a_{2}$, respectively, and $\alpha=\left(A, a_{1} / 2\right)$ with $A\left(a_{i}\right)=a_{i}, A\left(a_{5}\right)=-a_{5}, A\left(a_{6}\right)=-a_{6}, A\left(a_{7}\right)$ $=a_{1}+a_{2}+a_{3}+a_{4}-a_{7}$, and $A\left(a_{8}\right)=a_{2}-a_{8}$.

(2.4) $\Psi=\mathbf{Z}_{2}$ and $\Gamma$ is generated by $\left\{\alpha, t_{1}, \ldots, t_{8}\right\}$ where $\alpha^{2}=t_{1}, \alpha t_{i} \alpha^{-1}=t_{i}$, $\alpha t_{5} \alpha^{-1}=t_{5}^{-1}, \alpha t_{6} \alpha^{-1}=t_{1} t_{2} t_{3} t_{4} t_{6}^{-1}, \alpha t_{7} \alpha^{-1}=t_{2} t_{7}^{-1}, \alpha t_{8} \alpha^{-1}=t_{3} t_{8}^{-1} ;\left\langle a_{i}, a_{5}\right\rangle=0$, the projections of $a_{6}, a_{7}$ and $a_{8}$ on $E$ are $\frac{1}{2}\left(a_{1}+a_{2}+a_{3}+a_{4}\right), \frac{1}{2} a_{2}$, and $\frac{1}{2} a_{3}$ respectively, and $\alpha=\left(A, a_{1} / 2\right)$ with $A\left(a_{i}\right)=a_{i}, A\left(a_{5}\right)=-a_{5}, A\left(a_{6}\right)=a_{1}+a_{2}+a_{3}$ $+a_{4}-a_{6}, A\left(a_{7}\right)=a_{2}-a_{7}$, and $A\left(a_{8}\right)=a_{3}-a_{8}$.

(3.1) $\Psi=\mathbf{Z}_{3}$ and $\Gamma$ is generated by $\left\{\alpha, t_{1}, \ldots, t_{8}\right\}$ where $\alpha^{3}=t_{1}, \alpha t_{i} \alpha^{-1}=t_{i}$, $\alpha t_{5} \alpha^{-1}=t_{6}, \alpha t_{6} \alpha^{-1}=t_{5}^{-1} t_{6}^{-1}, \alpha t_{7} \alpha^{-1}=t_{8}, \alpha t_{8} \alpha^{-1}=t_{7}^{-1} t_{8}^{-1} ;\left\langle a_{i}, a_{j}\right\rangle=0,\left\{a_{5}, a_{6}\right\}$ and $\left\{a_{7}, a_{8}\right\}$ generate hexagonal plane lattices, and $\alpha=\left(A, a_{1} / 3\right)$ with $A\left(a_{i}\right)=a_{i}$, $A\left(a_{5}\right)=a_{6}, A\left(a_{6}\right)=-a_{5}-a_{6}, A\left(a_{7}\right)=a_{8}$, and $A\left(a_{8}\right)=-a_{7}-a_{8}$.

(3.2) $\Psi=\mathbf{Z}_{3}$ and $\Gamma$ is generated by $\left\{\alpha, t_{1}, \ldots, t_{8}\right\}$ where $\alpha^{3}=t_{1}, \alpha t_{i} \alpha^{-1}=t_{i}$, $\alpha t_{5} \alpha^{-1}=t_{6}, \quad \alpha t_{6} \alpha^{-1}=t_{5}^{-1} t_{6}^{-1}, \quad \alpha t_{7} \alpha^{-1}=t_{8}, \quad \alpha t_{8} \alpha^{-1}=t_{1} t_{2} t_{3} t_{4} t_{7}^{-1} t_{8}^{-1} ;\left\langle a_{i}, a_{5}\right\rangle=$ $\left\langle a_{i}, a_{6}\right\rangle=0,\left\{a_{5}, a_{6}\right\}$ generates a hexagonal plane lattice, the projection of $a_{7}$ and $a_{8}$ on $E$ is $\frac{1}{3}\left(a_{1}+a_{2}+a_{3}+a_{4}\right)$, and $\alpha=\left(A, a_{1} / 3\right)$ with $A\left(a_{i}\right)=a_{i}, A\left(a_{5}\right)=a_{6}$, $A\left(a_{6}\right)=-a_{5}-a_{6}, A\left(a_{7}\right)=a_{8}$, and $A\left(a_{8}\right)=a_{1}+a_{2}+a_{3}+a_{4}-a_{7}-a_{8}$.

(3.3) $\Psi=\mathbf{Z}_{3}$ and $\Gamma$ is generated by $\left\{\alpha, t_{1}, \ldots, t_{8}\right\}$ where $\alpha^{3}=t_{1}, \alpha t_{i} \alpha^{-1}=t_{i}$, $\alpha t_{5} \alpha^{-1}=t_{6}, \alpha t_{6} \alpha^{-1}=t_{1} t_{2} t_{3} t_{4} t_{5}^{-1} t_{6}^{-1}, \alpha t_{7} \alpha^{-1}=t_{8}, \alpha t_{8} \alpha^{-1}=t_{2} t_{7}^{-1} t_{8}^{-1} ;$ the projection of $a_{5}$ and $a_{6}$ on $E$ is $\frac{1}{3}\left(a_{1}+a_{2}+a_{3}+a_{4}\right)$, the projection of $a_{7}$ and $a_{8}$ on $E$ is $\frac{1}{3} a_{2}$, and $\alpha=\left(A, a_{1} / 3\right)$ with $A\left(a_{i}\right)=a_{i}, A\left(a_{5}\right)=a_{6}, A\left(a_{6}\right)=a_{1}+a_{2}+a_{3}+a_{4}-a_{5}$ $-a_{6}, A\left(a_{7}\right)=a_{8}$, and $A\left(a_{8}\right)=a_{2}-a_{7}-a_{8}$.

(4.1) $\Psi=\mathbf{Z}_{4}$ and $\Gamma$ is generated by $\left\{\alpha, t_{1}, \ldots, t_{8}\right\}$ where $\alpha^{4}=t_{1}, \alpha t_{i} \alpha^{-1}=t_{i}$, $\alpha t_{5} \alpha^{-1}=t_{6}, \alpha t_{6} \alpha^{-1}=t_{5}^{-1}, \alpha t_{7} \alpha^{-1}=t_{8}, \alpha t_{8} \alpha^{-1}=t_{7}^{-1} ;\left\langle a_{i}, a_{j}\right\rangle=0,\left\{a_{5}, a_{6}\right\}$ and 
$\left\{a_{7}, a_{8}\right\}$ generate a square plane lattice, and $\alpha=\left(A, a_{1} / 4\right)$ with $A\left(a_{i}\right)=a_{i}, A\left(a_{5}\right)=$ $a_{6}, A\left(a_{6}\right)=-a_{5}, A\left(a_{7}\right)=a_{8}$, and $A\left(a_{8}\right)=-a_{7}$.

(4.2) $\Psi=\mathbf{Z}_{4}$ and $\Gamma$ is generated by $\left\{\alpha, t_{1}, \ldots, t_{8}\right\}$ where $\alpha^{4}=t_{1}, \alpha t_{i} \alpha^{-1}=t_{i}$, $\alpha t_{5} \alpha^{-1}=t_{6}, \quad \alpha t_{6} \alpha^{-1}=t_{5}^{-1}, \quad \alpha t_{7} \alpha^{-1}=t_{8}, \quad \alpha t_{8} \alpha^{-1}=t_{1} t_{2} t_{3} t_{4} t_{7}^{-1} ;\left\langle a_{i}, a_{5}\right\rangle=$ $\left\langle a_{i}, a_{6}\right\rangle=0,\left\{a_{5}, a_{6}\right\}$ generates a square plane lattice, the projection of $a_{7}$ and $a_{8}$ on $E$ is $\frac{1}{2}\left(a_{1}+a_{2}+a_{3}+a_{4}\right)$, and $\alpha=\left(A, a_{1} / 4\right)$ with $A\left(a_{i}\right)=a_{i}, A\left(a_{5}\right)=a_{6}, A\left(a_{6}\right)=$ $-a_{5}, A\left(a_{7}\right)=a_{8}$, and $A\left(a_{8}\right)=a_{1}+a_{2}+a_{3}+a_{4}-a_{7}$.

(4.3) $\Psi=\mathbf{Z}_{4}$ and $\Gamma$ is generated by $\left\{\alpha, t_{1}, \ldots, t_{8}\right\}$ where $\alpha^{4}=t_{1}, \alpha t_{i} \alpha^{-1}=t_{i}$, $\alpha t_{5} \alpha^{-1}=t_{6}, \alpha t_{6} \alpha^{-1}=t_{1} t_{2} t_{3} t_{4} t_{5}^{-1}, \alpha t_{7} \alpha^{-1}=t_{8}, \alpha t_{8} \alpha^{-1}=t_{2} t_{7}^{-1}$; the projection of $a_{5}$ and $a_{6}$ on $E$ is $\frac{1}{2}\left(a_{1}+a_{2}+a_{3}+a_{4}\right)$, the projection of $a_{7}$ and $a_{8}$ on $E$ is $\frac{1}{2} a_{2}$, and $\alpha=\left(A, a_{1} / 4\right)$ with $A\left(a_{i}\right)=a_{i}, A\left(a_{5}\right)=a_{6}, A\left(a_{6}\right)=a_{1}+a_{2}+a_{3}+a_{4}-a_{5}$, $A\left(a_{7}\right)=a_{8}$, and $A\left(a_{8}\right)=a_{2}-a_{7}$.

(6) $\Psi=\mathbf{Z}_{6}$ and $\Gamma$ is generated by $\left\{\alpha, t_{1}, \ldots, t_{8}\right\}$ where $\alpha^{6}=t_{1}, \alpha t_{i} \alpha^{-1}=t_{i}$, $\alpha t_{5} \alpha^{-1}=t_{6}, \alpha t_{6} \alpha^{-1}=t_{5}^{-1} t_{6}, \alpha t_{7} \alpha^{-1}=t_{8}, \alpha t_{8} \alpha^{-1}=t_{7}^{-1} t_{8} ;\left\langle a_{i}, a_{j}\right\rangle=0,\left\{a_{5}, a_{6}\right\}$ and $\left\{a_{7}, a_{8}\right\}$ generate hexagonal plane lattices, and $\alpha=\left(A, a_{1} / 6\right)$ with $A\left(a_{i}\right)=a_{i}$, $A\left(a_{5}\right)=a_{6}, A\left(a_{6}\right)=a_{6}-a_{5}, A\left(a_{7}\right)=a_{8}$, and $A\left(a_{8}\right)=a_{8}-a_{7}$.

Proof. The proof consists of three parts. In part I, we show that each of the 12 types admits a quaternionic Kaehler structure (induced from $\mathbf{R}^{8}$ ). In part II, we show that no two of these types are affinely diffeomorphic. Finally, in part III, we show that any compact connected quaternionic Kaehler 8-manifold is affinely diffeomorphic to one of the 12 types.

I. First observe that each of the 12 types of $\Gamma \subset E(8)$ is a discrete uniform subgroup without elements of finite order. Hence $\mathbf{R}^{8} / \Gamma$ is a compact flat Riemannian manifold.

We must construct a quaternionic Kaehler structure $\{J, K\}$ on $\mathbf{R}^{8}$, invariant under $\Gamma$. Let $E$ be the span of $\left\{a_{1}, a_{2}, a_{3}, a_{4}\right\}$ and $E^{\perp}$ the orthogonal complement. Since $\mathbf{R}^{8}=E \oplus E^{\perp}$, we obtain the required structure by identifying each summand with $\mathbf{R}^{4}$ (provided with the standard quaternionic Kaehler structure). The identifications may be arbitrary except that we can assume $J$ preserves the span $E_{+}$of the projection of $\left\{a_{5}, a_{6}\right\}$ on $E^{\perp}$, and $\left\langle J a_{5}, a_{6}\right\rangle\left\langle J a_{7}, a_{8}\right\rangle<0$, where $\langle$,$\rangle is the$ standard inner product on Euclidean space.

To see that $\{J, K\}$ is invariant under $\Gamma$, it suffices to show that $A \in \Psi$ commutes with $\left.\{J, K\}\right|_{E}$ and $\{J, K\}_{E^{\perp}}$ (the standard structure on $\mathbf{R}^{4}$ is invariant under translation). This is obvious on $E$ for $\left.A\right|_{E}=$ Identity, and also on $E^{\perp}$ whenever $\left.A\right|_{E^{\perp}}= \pm$ Identity. A calculation shows that $\left.A\right|_{E_{+}}$is well-defined and is a rotation by $\frac{2 \pi}{3}, \frac{\pi}{2}, \frac{\pi}{3}$ depending on the holonomy type $3,4,6$, respectively. Since $\left.J\right|_{E_{+}}$and $\left.A\right|_{E_{+}}$are both rotations, they commute on $E_{+}$. Let $E_{-}$be the orthogonal complement, i.e., $E^{\perp}=E_{+}+E_{-}$. Since $A$ and $J$ are orthogonal, they both preserve $E_{-}$. A calculation shows that $\left.A\right|_{E_{-}}$is also a rotation by $\frac{2 \pi}{3}, \frac{\pi}{2}, \frac{\pi}{3}$ depending on the type. Hence, $A$ and $J$ commute on $E_{-}, E^{\perp}$, and finally $\mathbf{R}^{8}$.

We need to show that $A$ and $K$ commute; we will do this for holonomy type 4 and leave types 3 and 6 to the reader. If $X \in E_{+}$, then either $A X=J X$ or $A X=-J X$. We assume, without loss of generality, that $A X=J X$ (for we may replace $J$ by $-J$ everywhere). Let $X \in E_{+}$and $Y \in E_{-}$satisfy $K X=Y$. The condition 
$\left\langle J a_{5}, a_{6}\right\rangle\left\langle J a_{7}, a_{8}\right\rangle<0$ forces $A Y=-J Y$, and hence $A K X=-J K X=K J X=K A X$. A similar argument establishes the equality $A K=K A$ on $E_{-}$, so $A$ and $K$ commute.

II. Let $M=\mathbf{R}^{8} / \Gamma$, so that $\pi_{1}(M)=\Gamma$ and $H_{1}(M ; \mathbf{Z})=\Gamma /[\Gamma, \Gamma]$, where $[\Gamma, \Gamma]$ is the commutator subgroup generated by $[\Gamma, \Gamma]=\left\{\sigma \tau \sigma^{-1} \tau^{-1} \mid \sigma, \tau \in \Gamma\right\}$. In the table below, we record $H_{1}(M ; \mathbf{Z})$ for all 12 types; the calculations follow the table.

If $\mathbf{R}^{8} / \Gamma_{1}$ and $\mathbf{R}^{8} / \Gamma_{2}$ are affinely diffeomorphic, then $\Gamma_{1}$ and $\Gamma_{2}$ are isomorphic (Theorem 1). In particular, $\Gamma_{1}^{*} \simeq \Gamma_{2}^{*}$ (unique maximal abelian subgroups), $\Gamma_{1} / \Gamma_{1}^{*} \simeq$ $\Gamma_{2} / \Gamma_{2}^{*}$, and $\Gamma_{1} /\left[\Gamma_{1}, \Gamma_{1}\right] \simeq \Gamma_{2} /\left[\Gamma_{2} / \Gamma_{2}\right]$, i.e., the holonomy and first homology groups are isomorphic. From Table 1 , it then follows that $\mathbf{R}^{8} / \Gamma_{1}$ and $\mathbf{R}^{8} / \Gamma_{2}$ are of the same type.

TABLE 1

\begin{tabular}{|c|c||c|c|}
\hline Type & $H_{1}(M ; \mathbf{Z})$ & Type & $H_{1}(M ; \mathbf{Z})$ \\
\hline 1 & $\mathbf{Z}^{8}$ & 3.2 & $\mathbf{Z}^{4} \times \mathbf{Z}_{3}$ \\
2.1 & $\mathbf{Z}^{4} \times \mathbf{Z}_{2}^{4}$ & 3.3 & $\mathbf{Z}^{4}$ \\
2.2 & $\mathbf{Z}^{4} \times \mathbf{Z}_{2}^{3}$ & 4.1 & $\mathbf{Z}^{4} \times \mathbf{Z}_{2}^{2}$ \\
2.3 & $\mathbf{Z}^{4} \times \mathbf{Z}_{2}^{2}$ & 4.2 & $\mathbf{Z}^{4} \times \mathbf{Z}_{2}$ \\
2.4 & $\mathbf{Z}^{4} \times \mathbf{Z}_{2}$ & 4.3 & $\mathbf{Z}^{4}$ \\
3.1 & $\mathbf{Z}^{4} \times \mathbf{Z}_{3}^{2}$ & 6 & $\mathbf{Z}^{4}$ \\
\hline
\end{tabular}

(As usual, the subscript on $\mathbf{Z}$ is the modulus and the superscript is the multiplicity of the product.)

(1) $[\Gamma, \Gamma]$ is trivial, so $H_{1}=\Gamma=\mathbf{Z}^{8}$.

(2.1) $[\Gamma, \Gamma]$ is generated by $t_{k}^{2}$ for $k=5,6,7,8 ; H_{1}=\mathbf{Z}^{4} \times \mathbf{Z}_{2}^{4}$ with generators $\alpha[\Gamma, \Gamma]$ and $t_{k}[\Gamma, \Gamma]$ for $k=2,3, \ldots, 8$.

(2.2) $[\Gamma, \Gamma]$ is generated by $t_{k}^{2}$ for $k=5,6,7$, and $t_{1} t_{2} t_{3} t_{4} t_{8}^{-2}$; we may choose generators for $\Gamma$ as $\alpha, t_{1} t_{2} t_{3} t_{4} t_{8}^{-2}, t_{3}, t_{4}, \ldots, t_{8}$, and hence $H_{1}=\mathbf{Z}^{4} \times \mathbf{Z}_{2}^{3}$ with generators $\alpha[\Gamma, \Gamma]$ and $t_{k}[\Gamma, \Gamma]$ for $k=3,4, \ldots, 8$.

(2.3) $[\Gamma, \Gamma]$ is generated by $t_{5}^{2}, t_{6}^{2}, t_{1} t_{2} t_{3} t_{4} t_{7}^{-2}, t_{2} t_{8}^{-2}$; we may choose generators for $\Gamma$ as $\alpha, t_{1} t_{2} t_{3} t_{4} t_{7}^{-2}, t_{2} t_{8}^{-2}, t_{4}, \ldots, t_{8}$, and hence $H_{1}=\mathbf{Z}^{4} \times \mathbf{Z}_{2}^{2}$ with generators $\alpha[\Gamma, \Gamma]$ and $t_{k}[\Gamma, \Gamma]$ for $k=4, \ldots, 8$.

(2.4) $[\Gamma, \Gamma]$ is generated by $t_{5}^{2}, t_{1} t_{2} t_{3} t_{4} t_{6}^{-2}, t_{2} t_{7}^{-2}, t_{3} t_{8}^{-2}$; we may choose generators for $\Gamma$ as $\alpha, t_{1} t_{2} t_{3} t_{4} t_{6}^{-2}, t_{2} t_{7}^{-2}, t_{3} t_{8}^{-2}, t_{5}, t_{6}, t_{7}, t_{8}$, and hence $H_{1}=\mathbf{Z}^{4} \times \mathbf{Z}_{2}$ with generators $\alpha[\Gamma, \Gamma]$ and $t_{k}[\Gamma, \Gamma]$ for $k=5,6,7,8$.

(3.1) $[\Gamma, \Gamma]$ is generated by $t_{6} t_{5}^{-1}, t_{5} t_{6}^{2}, t_{8} t_{7}^{-1}, t_{7} t_{8}^{2}$, or equivalently by $t_{6} t_{5}^{-1}, t_{6}^{3}$, $t_{8} t_{7}^{-1}, t_{8}^{3}$; we may choose generators for $\Gamma$ as $\alpha, t_{1}, t_{2}, t_{3}, t_{4}, t_{6} t_{5}^{-1}, t_{6}, t_{8} t_{7}^{-1}, t_{8}$, and hence $H_{1}=\mathbf{Z}^{4} \times \mathbf{Z}_{3}^{2}$ with generators $\alpha[\Gamma, \Gamma]$ and $t_{k}[\Gamma, \Gamma]$ for $k=2,3,4,6,8$.

(3.2) $[\Gamma, \Gamma]$ is generated by $t_{6} t_{5}^{-1}, t_{5} t_{6}^{2}, t_{8} t_{7}^{-1}, t_{1} t_{2} t_{3} t_{4} t_{7}^{-1} t_{8}^{-2}$, or equivalently by $t_{6} t_{5}^{-1}, t_{6}^{3}, t_{8} t_{7}^{-1}, t_{1} t_{2} t_{3} t_{4} t_{8}^{-3}$; we may choose generators for $\Gamma$ as $\alpha, t_{1} t_{2} t_{3} t_{4} t_{8}^{-3}, t_{3}, t_{4}$, $t_{6} t_{5}^{-1}, t_{6}, t_{8} t_{7}^{-1}, t_{8}$, and hence $H_{1}=\mathbf{Z}^{4} \times \mathbf{Z}_{3}$ with generators $\alpha[\Gamma, \Gamma]$ and $t_{k}[\Gamma, \Gamma]$ for $k=3,4,6,8$.

(3.3) $[\Gamma, \Gamma]$ is generated by $t_{6} t_{5}^{-1}, t_{1} t_{2} t_{3} t_{4} t_{5}^{-1} t_{6}^{-2}, t_{8} t_{7}^{-1}, t_{2} t_{7}^{-1} t_{8}^{-2}$; we may choose generators for $\Gamma$ as $\alpha, t_{6} t_{5}^{-1}, t_{1} t_{2} t_{3} t_{4} t_{5}^{-1} t_{6}^{-2}, t_{8} t_{7}^{-1}, t_{2} t_{7}^{-1} t_{8}^{-2}, t_{4}, t_{6}, t_{8}$, and hence $H_{1}=\mathbf{Z}^{4}$ with generators $\alpha[\Gamma, \Gamma]$ and $t_{k}[\Gamma, \Gamma]$ for $k=4,6,8$. 
(4.1) $[\Gamma, \Gamma]$ is generated by $t_{6} t_{5}^{-1}, t_{5} t_{6}, t_{8} t_{7}^{-1}, t_{7} t_{8}$, or equivalently by $t_{6} t_{5}^{-1}, t_{6}^{2}$, $t_{8} t_{7}^{-1}, t_{8}^{2}$; we may choose generators for $\Gamma$ as $\alpha, t_{2}, t_{3}, t_{4}, t_{6} t_{5}^{-1}, t_{6}, t_{8} t_{7}^{-1}, t_{8}$, and hence $H_{1}=\mathbf{Z}^{4} \times \mathbf{Z}_{2}^{2}$ with generators $\alpha[\Gamma, \Gamma]$ and $t_{k}[\Gamma, \Gamma]$ for $k=2,3,4,6,8$.

(4.2) $[\Gamma, \Gamma]$ is generated by $t_{6} t_{5}^{-1}, t_{5} t_{6}, t_{8} t_{7}^{-1}, t_{1} t_{2} t_{3} t_{4} t_{7}^{-1} t_{8}^{-1}$, or equivalently by $t_{6} t_{5}^{-1}, t_{6}^{2}, t_{8} t_{7}^{-1}, t_{1} t_{2} t_{3} t_{4} t_{8}^{-2}$; we may choose generators for $\Gamma$ as $\alpha, t_{1} t_{2} t_{3} t_{4} t_{8}^{-2}, t_{3}, t_{4}$, $t_{6} t_{5}^{-1}, t_{6}, t_{8} t_{7}^{-1}, t_{8}$, and hence $H_{1}=\mathbf{Z}^{4} \times \mathbf{Z}_{2}$ with generators $\alpha[\Gamma, \Gamma]$ and $t_{k}[\Gamma, \Gamma]$ for $k=3,4,6,8$.

(4.3) $[\Gamma, \Gamma]$ is generated by $t_{6} t_{5}^{-1}, t_{1} t_{2} t_{3} t_{4} t_{5}^{-1} t_{6}^{-1}, t_{8} t_{7}^{-1}, t_{2} t_{7}^{-1} t_{8}^{-1}$; we may choose generators for $\Gamma$ as $\alpha, t_{6} t_{5}^{-1}, t_{1} t_{2} t_{3} t_{4} t_{5}^{-1} t_{6}^{-1}, t_{8} t_{7}^{-1}, t_{2} t_{7}^{-1} t_{8}^{-1}, t_{4}, t_{6}, t_{8}$, and hence $H_{1}=\mathbf{Z}^{4}$ with generators $\alpha[\Gamma, \Gamma]$ and $t_{k}[\Gamma, \Gamma]$ for $k=4,6,8$.

(6) $[\Gamma, \Gamma]$ is generated by $t_{6} t_{5}^{-1}, t_{5}, t_{8} t_{7}^{-1}, t_{7}$, or equivalently by $t_{5}, t_{6}, t_{7}, t_{8}$; hence $H_{1}=\mathbf{Z}_{4}$ with generators $\alpha[\Gamma, \Gamma]$ and $t_{k}[\Gamma, \Gamma]$ for $k=2,3,4$.

III. Let $M$ be a compact connected quaternionic Kaehler manifold. From $\S 1.1$ and Lemma 1, it follows that $M=\mathbf{R}^{8} / \Gamma$, where $\Gamma \subset E(8)$.

We begin by classifying $\Psi$. If $A \in \Psi$, then $A$ preserves the lattice $\Lambda$ (since $\Gamma^{*} \subset \Gamma$ is normal) and hence has a matrix representation with integral entries. In particular, the matrix invariants (trace, determinant, etc.) are integers. The vanishing of $\nabla J$ and $\nabla K$ implies $A J=J A$ and $A K=K A$.

Let $a \in \mathbf{R}^{8}$ be a nonzero vector such that $A(a)=a$, and let $E$ be the span of $\{a, J a, K a, J K a\}$ with 4-dimensional orthogonal complement $E^{\perp}$. Clearly $\left.A\right|_{E}=I$. Since $A, J$, and $K$ are orthogonal, $E^{\perp}$ is quaternionic and $\left.A\right|_{E^{\perp}}$ is well defined. If $\left.A\right|_{E^{\perp}} \neq I$, then $A-I: \mathbf{R}^{8} \rightarrow E^{\perp}$ is onto, and hence $\Lambda \cap E^{\perp}$ is a 4-dimensional lattice in $E^{\perp}$. In particular, $\left.A\right|_{E^{\perp}}$ has integral matrix invariants. Below, we will use the fact $\operatorname{tr}\left(\left.A\right|_{E^{\perp}}\right) \in \mathbf{Z}$.

If we fix the complex structure $J$, then $E^{\perp}$ becomes a 2-dimensional complex vector space and we may diagonalize $\left.A\right|_{E^{\perp}}$. Let $X \in E^{\perp}$ be a $J$-eigenvector with $J$-eigenvalue $\rho=\alpha+i \beta$, i.e., $A X=\alpha X+\beta J X$. The calculation $A K X=K A X=$ $\alpha K X-\beta J K X$ shows that $K X$ is also a $J$-eigenvector with $J$-eigenvalue $\bar{\rho}=\alpha-i \beta$. Since $A$ has finite order, say $n$, it follows that $\rho$ is a primitive $n$th root of unity. As a real $4 \times 4$ matrix relative to the basis $\{X, J X, K X, J K X\}$,

$$
\left.A\right|_{E^{\perp}}=\left[\begin{array}{llll}
\alpha & -\beta & 0 & 0 \\
\beta & \alpha & 0 & 0 \\
0 & 0 & \alpha & \beta \\
0 & 0 & -\beta & \alpha
\end{array}\right]
$$

with $\operatorname{tr}\left(\left.A\right|_{E^{\perp}}\right)=4 \alpha \in \mathrm{Z}$. By the algebraic proposition in $\S 3$, the only possible values for $n$ are $n=1,2,3,4$, and 6. In particular, we have $\rho= \pm 1, \pm i, e^{ \pm i \pi / 3}$, or $e^{ \pm i \pi / 6}$.

We can now complete the proof of

LeMma 2. The holonomy group $\Psi$ is either $\{1\}, \mathbf{Z}_{2}, \mathbf{Z}_{3}, \mathbf{Z}_{4}$, or $\mathbf{Z}_{6}$.

Proof. By the above remarks, it suffices to show that $\Psi$ is cyclic. We will first establish that $\Psi$ is simultaneously diagonalizable (or equivalently $\Psi$ is abelian), from which it easily follows that $\Psi$ is cyclic. 
Let $A, B \in \Psi$ be nontrivial. If $A B=I$, then $A=B^{-1}$ and $A B=B A . A B \neq I$ is harder. We will show (1) $A$ and $B$ fix a common 4-dimensional subspace $E$ (and hence the orthogonal complement $E^{\perp}$ ), and (2) $A$ and $B$ are simultaneously diagonalizable on these subspaces. Let $E_{C}$ denote the 4-dimensional +1 eigenspace of nontrivial $C \in \Psi$.

(1) If $X \in E_{A B} \cap B E_{A B}$ and $X \neq 0$, then $E_{A B}=B E_{A B}$, for $X, J X, K X, J K X$ generate $E_{A B}$ and the quaternionic structure commutes with $B$. If $B X=Y \in E_{A B}$, then $A Y=X \in E_{A B}$, and hence $E_{A B}=A E_{A B}$.

If $E_{A B} \cap B E_{A B}=0$, then we may write $Z \in E_{B A}$ as $Z=X_{1}+B X_{2}$ where $X_{1}$, $X_{2} \in E_{A B}$. Now, $Z=B A Z=B A X_{1}+B X_{2}$, and hence $X_{1}=B A X_{1}$, i.e., $E_{B A}=E_{A B}$. If $W \in E_{A}$, then we may write $W=Y_{1}+B Y_{2}$ where $Y_{1}, Y_{2} \in E_{A B}$. Now, $B W=$ $B A W=B A Y_{1}+B A B Y_{2}=Y_{1}+B Y_{2}$, and hence $W=B W$, i.e., $E_{A}=E_{B}=E_{A B}$.

(2) We will show that $A$ and $B$ commute on $E=E_{A}=E_{B}$ and $E^{\perp}$. This is trivial on $E$. On $E^{\perp}$, we may fix a complex structure, say $J$, and diagonalize

$$
\begin{array}{ll}
\left.A\right|_{E^{\perp}}=\left[\begin{array}{cc}
\rho & 0 \\
0 & \rho^{-1}
\end{array}\right] & \left.B\right|_{E^{\perp}}=\left[\begin{array}{cc}
\tau & 0 \\
0 & \tau^{-1}
\end{array}\right] \\
\operatorname{rel}\left\{X_{1}, X_{2}\right\} & \operatorname{rel}\left\{Y_{1}, Y_{2}\right\}
\end{array}
$$

where $\rho$ and $\tau$ are $J$-eigenvalues, the $J$-eigenvectors have unit length, and $X_{2}=K X_{1}$ and $Y_{2}=K Y_{1}$.

We have an $S^{2}$-action on $E^{\perp}$ given by $(a, b, c) \rightarrow a J+b K+c J K$, with $a^{2}+b^{2}$ $+c^{2}=1$. Recall that $F=a J+b K+c J K$ satisfies $F^{2}=-I$. For $X \in E^{\perp}$ and $\|X\|=1$, the orbit $S^{2} \cdot X$ is a standard 2-sphere of radius 1 . Since the dimension of $E^{\perp}$ is 4 , it follows that span $\left\{Y_{1}, J Y_{1}\right\}$ and $S^{2} \cdot X_{1}$ have a common element, which we may choose to be $Y_{1}=F X_{1}$. Now,

$$
\begin{gathered}
A Y_{1}=A F X_{1}=F A X_{1}=F \rho X_{1}=\rho^{-1} F X_{1}-2 \beta a X_{1}=\left(\rho^{-1}+2 \beta a F\right) Y_{1} \\
=\left[\alpha I+\left(2 \beta a^{2}-\beta\right) J+2 \beta a b K+2 \beta a c J K\right] Y_{1}
\end{gathered}
$$

and

$$
A Y_{1}=\tau Y_{1}=\gamma Y_{1}+\delta J Y_{1}
$$

where $\tau=\gamma+i \delta$. Thus

$$
\begin{gathered}
\alpha=\gamma, \\
2 \beta \alpha^{2}-\beta=\delta, \\
2 \beta \alpha b=0, \\
2 \beta a c=0 .
\end{gathered}
$$

If $\beta=0$, then $\delta=0$ and $\tau= \pm 1=\rho$, i.e., $\left.B\right|_{E^{\perp}}=I=\left.A\right|_{E^{\perp}}$ and they commute on $E^{\perp}$. If $a=0$, then $A Y_{1}=\rho^{-1} Y_{1}$, and hence $Y_{1}=\nu X_{2}$ for some $J$-complex number $\nu$. By orthogonality, $Y_{2}=\mu X_{1}$ for some $J$-complex number $\mu$, and it is clear that $A$ and $B$ commute on $E^{\perp}$. Finally, if $a \neq 0$ and $\beta \neq 0$, then $b=c=0$ and $F= \pm J$, i.e., $Y_{1}= \pm J X_{1}$. We may choose $Y_{2}$ so that $Y_{2}= \pm J X_{2}$, and it is clear that $A$ and $B$ commute on $E^{\perp}$. 
This completes the proofs of statements (1) and (2). We have shown that there is a decomposition $\mathbf{R}^{8}=E \oplus E^{\perp}$ and a $J$-complex basis $\left\{X_{1}, X_{2}\right\}$ for $E^{\perp}$ so that for any $A \in \Psi$, we have $\left.A\right|_{E}=I$ and

$$
\left.A\right|_{E^{\perp}}=\left[\begin{array}{cc}
\rho & 0 \\
0 & \rho^{-1}
\end{array}\right] .
$$

Furthermore, $\rho$ is an $n$th root of unity where $n=1,2,3,4$, or 6 . It follows easily that $\Psi=\{e\}, Z_{2}, Z_{3}, Z_{4}$, or $Z_{6}$. This completes the proof of the lemma. Q.E.D.

It remains to classify (up to an affine diffeomorphism commuting with $\Psi$ ) the lattices on $\mathbf{R}^{8}$ invariant under each $\Psi$ :

$\Psi=\{e\}$.

In this case, $\Gamma=\Gamma^{*}$ and $\mathbf{R}^{8} / \Gamma$ is a torus. Clearly any two lattices are invariant and equivalent under $\Psi$, and any two tori are affinely diffeomorphic.

$\Psi=\mathbf{Z}_{2}$.

Choose $(A, a) \in \Gamma$ such that $A$ generates $\Psi, A(a)=a$, and $\|a\|$ minimal for this. Note that $a$ is not in the lattice $\Lambda$. Otherwise, $(I,-a) \in \Gamma$ and $(A, a)(I,-a)=$ $(A, 0) \in \Gamma$; but $(A, 0)$ has 0 as a fixed point; contradiction. For $\Psi=\mathbf{Z}_{2}$, we have $\left.A\right|_{E^{\perp}}=-I$ and $A+I: \mathbf{R}^{8} \rightarrow E$. Since $A$ preserves $\Lambda$, it follows that $\Lambda \cap E$ is a 4-dimensional lattice with generators, say $a_{1}, a_{2}, a_{3}$, and $a_{4}$. Now $(A, a)^{2}=(I, 2 a)$ shows that $2 a \in \Lambda \cap E$, and by the minimality of $\|a\|$, we may choose these generators so that $a=a_{1} / 2$. If we complete $\left\{a_{1}, a_{2}, a_{3}, a_{4}\right\}$ to a full set of lattice generators for $\Lambda$, by adding $a_{5}, a_{6}, a_{7}$, and $a_{8}$, then we have

$$
a_{j}=\alpha_{j}^{1} a_{1}+\alpha_{j}^{2} a_{2}+\alpha_{j}^{3} a_{3}+\alpha_{j}^{4} a_{4}+\beta_{j} b_{j}
$$

where $j=5,6,7,8, b_{j} \in E^{\perp}$, and the coefficients are real numbers. Since $A\left(a_{j}\right)+$ $a_{j}=2 \alpha_{j}^{1} a_{1}+\cdots+2 \alpha_{j}^{4} a_{4} \in \Lambda$, we may reduce $\bmod \left\{a_{1}, a_{2}, a_{3}, a_{4}\right\}$ to see that $\alpha_{j}^{i}$ is 0 or $\frac{1}{2}$, for $i=1,2,3,4$ and $j=5,6,7,8$.

For $j=5,6,7,8$, we will examine the possible 4-tuples $\left(\alpha_{j}^{1}, \alpha_{j}^{2}, \alpha_{j}^{3}, \alpha_{j}^{4}\right)$ whose entries are either 0 or $\frac{1}{2}$. The easiest case is $\alpha_{j}^{i}=0$ for all $i$ and $j$, that is, $a_{j} \in E^{\perp}$ and $A\left(a_{j}\right)=-a_{j}$. Hence $(A, a)$ and $\left(I, a_{k}\right), k=2,3, \ldots, 8$, generate $\Gamma$ and the relations are given by type 2.1 (the quaternionic Klein bottle).

Assume that $a_{5}, a_{6}, a_{7} \in E^{\perp}$, i.e., $\alpha_{5}^{i}=\alpha_{6}^{i}=\alpha_{7}^{i}=0$ for $i=1,2,3,4$, and $a_{8} \notin E^{\perp}$. If $\alpha_{8}^{i}=\frac{1}{2}$ for exactly one $i$, then by renumbering, we may assume that $\alpha_{8}^{1}=\frac{1}{2}$ or $\alpha_{8}^{2}=\frac{1}{2}$ (note that $a_{1}$ is distinguished in $\left\{a_{1}, a_{2}, a_{3}, a_{4}\right\}$ by the requirement $\left.a=a_{1} / 2\right)$. If $\left(\alpha_{8}^{1}, \alpha_{8}^{2}, \alpha_{8}^{3}, \alpha_{8}^{4}\right)=\left(\frac{1}{2}, 0,0,0\right)$, that is, $a_{8}=\frac{1}{2} a_{1}+\beta_{8} b_{8}$, then $\left(I,-a_{8}\right)(A, a)=\left(A, a-a_{8}\right)=\left(A,-\beta_{8} b_{8}\right)$ and this rigid motion fixes $-\frac{1}{2} \beta_{8} b_{8}$; contradiction. For $\alpha_{8}^{2}=\frac{1}{2}$, we change the lattice generator $a_{2}$ by $a_{2} \rightarrow a_{2}-a_{1}-a_{3}$ $-a_{4}$ to obtain $\alpha_{8}^{i}=\frac{1}{2}$ for all $i$. In this way we see that if one or more of $\alpha_{8}^{i}$ equal $\frac{1}{2}$, then either there is a contradiction or, by a suitable change of the lattice generators in $E$, we may assume $a_{8}=\frac{1}{2} a_{1}+\frac{1}{2} a_{2}+\frac{1}{2} a_{3}+\frac{1}{2} a_{4}+\beta_{8} b_{8}$, where $b_{8} \in E$. Hence $A\left(a_{8}\right)=\frac{1}{2} a_{1}+\cdots+\frac{1}{2} a_{4}-\beta_{8} b_{8}=a_{1}+\cdots+a_{4}-a_{8}$ and this flat manifold is of type 2.2 .

Assume that $a_{5}, a_{6} \in E^{\perp}$, i.e., $\alpha_{5}^{i}=\alpha_{6}^{i}=0$ for $i=1,2,3,4$, and $a_{7}, a_{8} \notin E^{\perp}$. By the above argument, we may assume $\left(\alpha_{7}^{1}, \alpha_{7}^{2}, \alpha_{7}^{3}, \alpha_{7}^{4}\right)=\left(\frac{1}{2}, \frac{1}{2}, \frac{1}{2}, \frac{1}{2}\right)$, in particular, 
$A\left(a_{7}\right)=a_{1}+\cdots+a_{4}-a_{7}$. Below we list the possibilities for $\left(\alpha_{8}^{1}, \alpha_{8}^{2}, \alpha_{8}^{3}, \alpha_{8}^{4}\right)$, up to a permutation of $\left\{a_{2}, a_{3}, a_{4}\right\}$ (always $a_{1}$ is fixed by the condition $a=a_{1} / 2$ ). Most of the entries reduce to the third entry by a change of lattice generators; we indicate this change next to each 4-tuple. Two of the entries give contradictions for we cannot obtain a lattice vector with associated 4-tuple $\left(\frac{1}{2}, 0,0,0\right)$ (as mentioned above this contradicts the freeness of the $\Gamma$-action). The last entry may be reduced to type 2.2 by the indicated change of lattice vector. In changing generators, we must be careful to preserve the assumption $\alpha_{7}^{i}=\frac{1}{2}$, for all $i$, or equivalently, the sum $a_{1}+a_{2}+a_{3}+a_{4}$. If a lattice generator does not appear, then it is left unchanged.

$$
\begin{array}{ll}
(0,0,0,0) & a_{8} \rightarrow a_{7}, a_{7} \rightarrow a_{8} \text { (reduces to type 2.2) } \\
\left(\frac{1}{2}, 0,0,0\right) & \text { contradiction } \\
\left(0, \frac{1}{2}, 0,0\right) & \text { type } 2.3 \\
\left(\frac{1}{2}, \frac{1}{2}, 0,0\right) & a_{2} \rightarrow a_{2}+a_{1}, a_{3} \rightarrow a_{3}-a_{1} \text { (reduces to type 2.3) } \\
\left(0, \frac{1}{2}, \frac{1}{2}, 0\right) & a_{2} \rightarrow a_{2}+a_{3}, a_{4} \rightarrow a_{4}-a_{3} \text { (reduces to type 2.3) } \\
\left(\frac{1}{2}, \frac{1}{2}, \frac{1}{2}, 0\right) & a_{2} \rightarrow a_{2}+a_{1}+a_{3}, a_{4} \rightarrow a_{4}-a_{1}-a_{3} \text { (reduces to type 2.3) } \\
\left(0, \frac{1}{2}, \frac{1}{2}, \frac{1}{2}\right) & a_{7}-a_{8} \text { gives }\left(\frac{1}{2}, 0,0,0\right) ; \text { contradiction } \\
\left(\frac{1}{2}, \frac{1}{2}, \frac{1}{2}, \frac{1}{2}\right) & a_{7} \rightarrow a_{7}-a_{8} \text { (reduces to type 2.2) }
\end{array}
$$

For those entries that reduce to $\left(0, \frac{1}{2}, 0,0\right)$, we have $a_{8}=\frac{1}{2} a_{2}+\beta_{8} b_{8}$ and $A\left(a_{8}\right)$ $=\frac{1}{2} a_{2}-\beta_{8} b_{8}=a_{2}-a_{8}$; this is of type 2.3.

Assume now that $a_{5} \in E^{\perp}$, i.e., $\alpha_{5}^{i}=0$ for $i=1,2,3,4$, and $a_{6}, a_{7}, a_{8} \notin E^{\perp}$. The above argument shows that we may assume $\left(\alpha_{6}^{1}, \alpha_{6}^{2}, \alpha_{6}^{3}, \alpha_{6}^{4}\right)=\left(\frac{1}{2}, \frac{1}{2}, \frac{1}{2}, \frac{1}{2}\right)$ and $\left(\alpha_{7}^{1}, \alpha_{7}^{2}, \alpha_{7}^{3}, \alpha_{7}^{4}\right)=\left(0, \frac{1}{2}, 0,0\right)$. Below we list the possible 4-tuples for $a_{8}$, up to a permutation of $\left\{a_{3}, a_{4}\right\}$. In changing generators, we must preserve $a_{1}, a_{2}$, and $a_{1}+\cdots+a_{4}$.

$$
\begin{array}{ll}
(0,0,0,0) & \left.a_{6} \rightarrow a_{7}, a_{7} \rightarrow a_{8}, a_{8} \rightarrow a_{6} \text { (reduces to type } 2.3\right) \\
\left(\frac{1}{2}, 0,0,0\right) & \text { contradiction } \\
\left(0, \frac{1}{2}, 0,0\right) & a_{8} \rightarrow a_{8}-a_{7} \text { (reduces to type 2.3) } \\
\left(0,0, \frac{1}{2}, 0\right) & \text { type } 2.4 \\
\left(\frac{1}{2}, \frac{1}{2}, 0,0\right) & a_{8}-a_{7} \text { gives }\left(\frac{1}{2}, 0,0,0\right) ; \text { contradiction } \\
\left(\frac{1}{2}, 0, \frac{1}{2}, 0\right) & \left.a_{3} \rightarrow a_{3}+a_{1}, a_{4} \rightarrow a_{4}-a_{1} \text { (reduces to type } 2.3\right) \\
\left(0, \frac{1}{2}, \frac{1}{2}, 0\right) & a_{3} \rightarrow a_{3}+a_{2}, a_{4} \rightarrow a_{4}-a_{2}(\text { reduces to type } 2.3) \\
\left(0,0, \frac{1}{2}, \frac{1}{2}\right) & a_{6}-a_{7}-a_{8} \text { gives }\left(\frac{1}{2}, 0,0,0\right) ; \text { contradiction } \\
\left(\frac{1}{2}, \frac{1}{2}, \frac{1}{2}, 0\right) & \left.a_{3} \rightarrow a_{3}+a_{2}+a_{1}, a_{4} \rightarrow a_{4}-a_{2}-a_{1} \text { (reduces to type } 2.3\right) \\
\left(\frac{1}{2}, 0, \frac{1}{2}, \frac{1}{2}\right) & a_{8} \rightarrow a_{6}-a_{7}-a_{8}(\text { reduces to type 2.2) } \\
\left(0, \frac{1}{2}, \frac{1}{2}, \frac{1}{2}\right) & a_{6}-a_{8} \text { gives }\left(\frac{1}{2}, 0,0,0\right) ; \text { contradiction } \\
\left(\frac{1}{2}, \frac{1}{2}, \frac{1}{2}, \frac{1}{2}\right) & a_{8} \rightarrow a_{8}-a_{6} \text { (reduces to type 2.2) }
\end{array}
$$

The fourth entry in this list gives the only new manifold. It is characterized by $A\left(a_{8}\right)=\frac{1}{2} a_{3}-\beta_{8} b_{8}=a_{3}-a_{8}$ where $a_{8}=\frac{1}{2} a_{3}+\beta_{8} b_{8}$; this is of type 2.4 . 
Finally, by the above arguments, we may assume $\left(\alpha_{5}^{1}, \alpha_{5}^{2}, \alpha_{5}^{3}, \alpha_{5}^{4}\right)=\left(\frac{1}{2}, \frac{1}{2}, \frac{1}{2}, \frac{1}{2}\right)$, $\left(\alpha_{6}^{1}, \alpha_{6}^{2}, \alpha_{6}^{3}, \alpha_{6}^{4}\right)=\left(0, \frac{1}{2}, 0,0\right)$, and $\left(\alpha_{7}^{1}, \alpha_{7}^{2}, \alpha_{7}^{3}, \alpha_{7}^{4}\right)=\left(0,0, \frac{1}{2}, 0\right)$. The possibilities for $a_{8}$ are listed below. The generator changes must preserve $a_{1}, a_{2}, a_{3}$, and $a_{4}$.

$\begin{array}{ll}(0,0,0,0) & a_{5} \rightarrow a_{6}, a_{6} \rightarrow a_{7}, a_{7} \rightarrow a_{8}, a_{8} \rightarrow a_{5} \quad \text { (reduces to type 2.4) } \\ \left(\frac{1}{2}, 0,0,0\right) & \text { contradiction } \\ \left(0, \frac{1}{2}, 0,0\right) & \left.a_{8} \rightarrow a_{6}-a_{8} \quad \text { (reduces to type } 2.4\right) \\ \left(0,0, \frac{1}{2}, 0\right) & \left.a_{8} \rightarrow a_{7}-a_{8} \quad \text { (reduces to type } 2.4\right) \\ \left(0,0,0, \frac{1}{2}\right) & a_{5}-a_{6}-a_{7}-a_{8} \text { gives }\left(\frac{1}{2}, 0,0,0\right) ; \text { contradiction } \\ \left(\frac{1}{2}, \frac{1}{2}, 0,0\right) & a_{8}-a_{6} \text { gives }\left(\frac{1}{2}, 0,0,0\right) ; \text { contradiction } \\ \left(\frac{1}{2}, 0, \frac{1}{2}, 0\right) & a_{8}-a_{7} \text { gives }\left(\frac{1}{2}, 0,0,0\right) ; \text { contradiction } \\ \left(\frac{1}{2}, 0,0, \frac{1}{2}\right) & \left.a_{8} \rightarrow a_{5}-a_{6}-a_{7}-a_{8} \text { (reduces to type } 2.4\right) \\ \left(0, \frac{1}{2}, \frac{1}{2}, 0\right) & \left.a_{8} \rightarrow a_{8}-a_{6}-a_{7} \text { (reduces to type } 2.4\right) \\ \left(0, \frac{1}{2}, 0, \frac{1}{2}\right) & a_{8} \rightarrow a_{5}-a_{7}-a_{8} \text { gives }\left(\frac{1}{2}, 0,0,0\right) ; \text { contradiction } \\ \left(0,0, \frac{1}{2}, \frac{1}{2}\right) & a_{8} \rightarrow a_{5}-a_{6}-a_{8} \text { gives }\left(\frac{1}{2}, 0,0,0\right) ; \text { contradiction } \\ \left(\frac{1}{2}, \frac{1}{2}, \frac{1}{2}, 0\right) & a_{8} \rightarrow a_{8}-a_{6}-a_{7} \text { gives }\left(\frac{1}{2}, 0,0,0\right) ; \text { contradiction } \\ \left(\frac{1}{2}, \frac{1}{2}, 0, \frac{1}{2}\right) & \left.a_{8} \rightarrow a_{5}-a_{7}-a_{8} \text { (reduces to type } 2.4\right) \\ \left(\frac{1}{2}, 0, \frac{1}{2}, \frac{1}{2}\right) & \left.a_{8} \rightarrow a_{5}-a_{6}-a_{8} \text { (reduces to type } 2.4\right) \\ \left(0, \frac{1}{2}, \frac{1}{2}, \frac{1}{2}\right) & \left.a_{8} \rightarrow a_{5}-a_{8} \text { gives ( } \frac{1}{2}, 0,0,0\right) ; \text { contradiction } \\ \left(\frac{1}{2}, \frac{1}{2}, \frac{1}{2}, \frac{1}{2}\right) & a_{8} \rightarrow a_{5}-a_{8} \text { (reduces to type 2.4) }\end{array}$

In this list, no new types appear. In particular, we may always choose one of the lattice generators to be in $E^{\perp}$. This completes the classification with holonomy $\mathbf{Z}_{2}$. $\Psi=\mathbf{Z}_{3}$.

Choose $(A, a) \in \Gamma$ such that $A$ generates $\Psi, A(a)=a$, and $\|a\|$ minimal for this. As noted in the $\mathbf{Z}_{2}$ case, $a \notin \Lambda$. We again fix $J$ on $E^{\perp}$ and diagonalize

$$
\left.A\right|_{E^{\perp}}=\left[\begin{array}{cc}
\rho & 0 \\
0 & \rho^{-1}
\end{array}\right]
$$

where $\rho$ is a cube root of unity. Hence $A^{2}+A+I: \mathbf{R}^{8} \rightarrow E$ preserves $A$, and it follows that $\Lambda \cap E$ is a 4-dimensional lattice with generators $a_{1}, a_{2}, a_{3}$, and $a_{4}$. Now $(A, a)^{3}=(I, 3 a)$ shows that $3 a \in \Lambda$, and by the minimality of $\|a\|$, we may choose these generators so that $a=a_{1} / 3$.

To complete the set of lattice generators, we will use the following lemma.

LEMMA 3. For $\Psi=\mathbf{Z}_{3}$, there is a set of lattice generators $\left\{a_{1}, \ldots, a_{8}\right\}$ for $\Lambda$ satisfying $a_{1}, a_{2}, a_{3}, a_{4} \in E, A\left(a_{5}\right)=a_{6}$, and $A\left(a_{7}\right)=a_{8}$.

Proof. It suffices to choose $a_{5}, a_{6}, a_{7}, a_{8}$ satisfying the above condition. Let $P_{V}$ be the projection onto the vector subspace $V \subset \mathbf{R}^{8}$. Choose $a_{5} \notin E \cap \Lambda$ which minimizes the length $\left\|P_{E^{\perp}} a_{5}\right\|$ among all nonzero lattice vectors not in $E \cap \Lambda$ (the discreteness of $\Lambda$ allows the minimum). Let $\Lambda_{1}$ be the sublattice generated by $\left\{a_{1}\right.$, $\left.a_{2}, a_{3}, a_{4}, a_{5}, A\left(a_{5}\right)\right\}$, and let $E_{1}$ be the span of these vectors. Choose $a_{7} \notin \Lambda_{1}$ which minimizes the length $\left\|P_{E_{1}^{\perp}} a_{7}\right\|$ among all nonzero lattice vectors not in $\Lambda_{1}$. 
We claim that $S=\left\{a_{1}, \ldots, a_{5}, A\left(a_{5}\right), a_{7}, A\left(a_{7}\right)\right\}$ generates $\Lambda$. To see this, assume the existence of a lattice vector $y$ not in the integral span of $S$. It is easy to see that $S$ spans $\mathbf{R}^{\mathbf{8}}$, and we may write

$$
y=r_{1} a_{1}+\cdots+r_{5} a_{5}+r_{6} A\left(a_{5}\right)+r_{7} a_{7}+r_{8} A\left(a_{7}\right),
$$

where at least one of the coefficients is not integral. By adding integral multiples of $S$, we may assume that $\left|r_{i}\right| \leqslant \frac{1}{2}, i=1, \ldots, 8$. Also we may assume $y \notin E \cap \Lambda$ since $\left\{a_{1}, a_{2}, a_{3}, a_{4}\right\}$ were chosen to generate $E \cap \Lambda$. Hence, at least one of $r_{5}, r_{6}, r_{7}, r_{8}$ is nonzero. If $r_{7}$ or $r_{8}$ is nonzero, then $y \notin \Lambda_{1}$ and

$$
P_{E_{1}^{\perp}} y=r_{7} p_{E_{1}^{\perp}} a_{7}+r_{8} P_{E_{1}^{\perp}} A\left(a_{7}\right)
$$

and

$$
\left\|P_{E_{1}^{\perp}} y\right\|=\left(r_{7}^{2}+2 r_{7} r_{8} \cos ( \pm \pi / 3)+r_{8}^{2}\right)^{\frac{1}{2}}\left\|P_{E_{1}^{\perp}} a_{7}\right\|<\left\|P_{E_{1}^{\perp}} a_{7}\right\| .
$$

This contradicts our choice of $a_{7}$. If $r_{7}$ and $r_{8}$ are both zero, then

$$
P_{E^{\perp}} y=r_{5} P_{E^{\perp}} a_{5}+r_{6} P_{E^{\perp}} A\left(a_{5}\right)
$$

and, as above,

$$
\left\|P_{E^{\perp}} y\right\|<\left\|P_{E^{\perp}} a_{5}\right\| .
$$

This contradicts our choice of $a_{5}$, and hence $y$ does not exist. Q.E.D.

Let us choose generators for $\Lambda$ from Lemma 3 and set

$$
a_{j}=\alpha_{j}^{1} a_{1}+\alpha_{j}^{2} a_{2}+\alpha_{j}^{3} a_{3}+\alpha_{j}^{4} a_{4}+\beta_{j} b_{j}
$$

where $j=5$ or 7 ( $a_{6}$ and $a_{8}$ are determined by Lemma 3 ), $b_{j} \in E$, and the coefficients are real numbers. Since $A^{2}\left(a_{j}\right)+A\left(a_{j}\right)+a_{j}=3 \alpha_{j}^{1} a_{1}+\cdots+3 \alpha_{j}^{4} a_{4} \in$ $\Lambda$, we may reduce $\bmod \left\{a_{1}, a_{2}, a_{3}, a_{4}\right\}$ to see that the only possibilities for $\alpha_{j}^{i}$ are 0 , $\frac{1}{3}, \frac{2}{3}$, where $i=1,2,3,4$ and $j=5,7$.

For $j=5$, 7, we will examine the possible 4-tuples $\left(\alpha_{j}^{1}, \alpha_{j}^{2}, \alpha_{j}^{3}, \alpha_{j}^{4}\right)$ whose entries are either $0, \frac{1}{3}$, or $\frac{2}{3}$. The easiest case is $\alpha_{j}^{i}=0$ for $i=1,2,3,4$ and $j=5$, 7, i.e., $a_{j} \in E, A\left(a_{j}\right)=a_{j+1}$, and $A\left(a_{j+1}\right)=-a_{j}-a_{j+1}$ for $j=5$, 7. Hence $(A, a)$ and $\left(I, a_{k}\right), k=2, \ldots, 8$, generate $\Gamma$ and the relations are given by type 3.1 .

Assume that $a_{5} \in E^{\perp}$, i.e., $\alpha_{5}^{i}=\alpha_{6}^{i}=0$ for $i=1,2,3,4$, and $a_{7} \notin E^{\perp}$. If $\alpha_{7}^{i} \in\left\{0, \frac{1}{3}\right\}$ for all $i$, then by an argument analogous to the $\mathbf{Z}_{2}$ case, we choose $\left\{a_{1}\right.$, $\left.a_{2}, a_{3}, a_{4}\right\}$ so that $\alpha_{7}^{i}=\frac{1}{3}$ for all $i$ (similarly if $\alpha_{7}^{i} \in\left\{0, \frac{2}{3}\right\}$ ). If $\alpha_{7}^{i}=\frac{2}{3}$ for all $i$, then the change of basis $a_{7} \rightarrow a_{1}+a_{2}+a_{3}+a_{4}-a_{7}$ gives $\alpha_{7}^{i}=\frac{1}{3}$ for all $i$ (we must change $a_{8}$ accordingly). It is obvious that the new basis will generate $\Lambda$.

If both $\frac{1}{3}$ and $\frac{2}{3}$ appear in $\left(\alpha_{7}^{1}, \alpha_{7}^{2}, \alpha_{7}^{3}, \alpha_{7}^{4}\right)$, we have 3 possibilities: $\left(\frac{1}{3}, \frac{2}{3}, *, *\right)$, $\left(\frac{2}{3}, \frac{1}{3}, *, *\right)$ or $\left(*, \frac{1}{3}, \frac{2}{3}, *\right)$, where $* \in\left\{0, \frac{1}{3}, \frac{2}{3}\right\}$ and no two $*$ are necessarily equal. The first 4-tuple becomes $\left(\frac{1}{3}, \frac{1}{3}, *, *\right)$ by the change $a_{2} \rightarrow a_{1}+a_{2}$ and $a_{7} \rightarrow a_{1}+a_{2}$ $-a_{7}$. The second becomes $\left(\frac{1}{3}, \frac{1}{3}, *, *\right)$ by the change $a_{2} \rightarrow a_{1}+a_{2}$, and the third becomes $\left(*, \frac{1}{3}, \frac{1}{3}, *\right)$ by the change $a_{2} \rightarrow a_{2}+a_{3}$. It is clear from this argument that we may eliminate all $\frac{2}{3}$ 's. 
So for $a_{5} \in E^{\perp}$ and $a_{7} \notin E^{\perp}$, we may change the lattice generators (preserving $a_{1}$ and satisfying the conditions in Lemma 3 ) so that $\alpha_{7}^{i}=\frac{1}{3}$ for all $i$, i.e.,

$$
\begin{aligned}
a_{7}= & \frac{1}{3} a_{1}+\cdots+\frac{1}{3} a_{4}+\beta_{7} b_{7}, \quad b_{7} \in E^{\perp}, \\
\left(a_{7}\right) & =\frac{1}{3} a_{1}+\cdots+\frac{1}{3} a_{4}+\beta_{7} A\left(b_{7}\right), \\
A\left(a_{8}\right) & =A^{2}\left(a_{7}\right)=\frac{1}{3} a_{1}+\cdots+\frac{1}{3} a_{4}+\beta_{7} A^{2}\left(b_{7}\right) \\
& =\frac{1}{3} a_{1}+\cdots+\frac{1}{3} a_{4}-\beta_{7} b_{7}-\beta_{7} A\left(b_{7}\right) \\
& =a_{1}+\cdots+a_{4}-a_{7}-a_{8} .
\end{aligned}
$$

This is of type 3.2.

Finally, we assume that $a_{5}, a_{7} \notin E^{\perp}$. By the above argument, we may assume $\alpha_{5}^{i}=\frac{1}{3}$ for all $i$, i.e., $a_{5}=\frac{1}{3} a_{1}+\cdots+\frac{1}{3} a_{4}+\beta_{5} b_{5}$. For $\left(\alpha_{7}^{1}, \alpha_{7}^{2}, \alpha_{7}^{3}, \alpha_{7}^{4}\right)$, we shall reduce each possibility to $\left(0, \frac{1}{3}, 0,0\right)$ by a suitable change of lattice generators (preserving $a_{1}$ and $a_{1}+\cdots+a_{4}$, and satisfying the conditions in Lemma 3 ). If $\alpha_{7}^{i} \in\left\{0, \frac{1}{3}\right\}$ for all $i$, then the arguments in the $\mathbf{Z}_{2}$ case show that we may reduce $\left(\alpha_{7}^{1}, \alpha_{7}^{2}, \alpha_{7}^{3}, \alpha_{7}^{4}\right)$ to $\left(0, \frac{1}{3}, 0,0\right)$. If $\alpha_{7}^{i} \in\left\{0, \frac{2}{3}\right\}$ for all $i$, we may replace $\frac{2}{3}$ by $\frac{1}{3}$ as the following example illustrates: $\left(\frac{2}{3}, \frac{2}{3}, 0,0\right)$ becomes $\left(\frac{1}{3}, \frac{1}{3}, 0,0\right)$ by the change $a_{7} \rightarrow a_{1}$ $+a_{2}-a_{7}$. If both $\frac{1}{3}$ and $\frac{2}{3}$ appear, and if $\frac{2}{3}$ occurs more often than $\frac{1}{3}$, we may reverse the roles of $\frac{1}{3}$ and $\frac{2}{3}$ by $a_{7} \rightarrow \delta_{1} a_{1}+\delta_{2} a_{2}+\delta_{3} a_{3}+\delta_{4} a_{4}-a_{7}$, where $\delta_{i}=0$ or 1 whenever $\alpha_{7}^{i}=0$ or $\frac{2}{3}$ respectively. With these simplifications, we list below, up to a permutation of $\left\{a_{2}, a_{3}, a_{4}\right\}$, the possibilities for $\left(\alpha_{7}^{1}, \alpha_{7}^{2}, \alpha_{7}^{3}, \alpha_{7}^{4}\right)$. The given lattice changes replace the given 4 -tuple with $\left(0, \frac{1}{3}, 0,0\right)$.

$$
\begin{array}{ll}
\left(0, \frac{1}{3}, \frac{2}{3}, 0\right) & a_{2} \rightarrow a_{2}+2 a_{3}, a_{4} \rightarrow a_{4}-2 a_{3} \text { (reduces to type 3.3) } \\
\left(\frac{1}{3}, \frac{2}{3}, 0,0\right) & a_{2} \rightarrow a_{2}+2 a_{1}, a_{3} \rightarrow a_{3}-2 a_{1}, a_{7} \rightarrow a_{1}+a_{2}-a_{7} \\
& \text { (reduces to type 3.3) } \\
\left(0, \frac{2}{3}, \frac{1}{3}, \frac{1}{3}\right) & \left.a_{2} \rightarrow a_{2}-a_{1}, a_{3} \rightarrow a_{3}+a_{1}, a_{7} \rightarrow a_{7}-a_{5} \text { (reduces to type } 3.3\right) \\
\left(\frac{1}{3}, \frac{1}{3}, \frac{2}{3}, 0\right) & a_{2} \rightarrow a_{2}+a_{1}+2 a_{3}, a_{4} \rightarrow a_{4}-a_{1}-2 a_{3} \text { (reduces to type 3.3) } \\
\left(\frac{2}{3}, \frac{1}{3}, \frac{1}{3}, 0\right) & a_{2} \rightarrow a_{2}+2 a_{1}+a_{3}, a_{4} \rightarrow a_{4}-2 a_{1}-a_{3} \text { (reduces to type 3.3) } \\
\left(\frac{2}{3}, \frac{2}{3}, \frac{1}{3}, \frac{1}{3}\right) & a_{2} \rightarrow a_{2}+a_{1}, a_{3} \rightarrow a_{3}-a_{1}, a_{7} \rightarrow a_{7}-a_{5} \text { (reduces to type 3.3) } \\
\left(\frac{1}{3}, \frac{2}{3}, \frac{1}{3}, \frac{1}{3}\right) & a_{7} \rightarrow a_{7}-a_{5}(\text { reduces to type 3.3) } \\
\left(\frac{2}{3}, \frac{1}{3}, \frac{1}{3}, \frac{1}{3}\right) & a_{7}-a_{5} \text { gives }\left(\frac{1}{3}, 0,0,0\right) ; \text { contradiction }
\end{array}
$$

Hence, for $a_{5}, a_{7} \notin E^{\perp}$, we may change the lattice generators so that $\left(\alpha_{5}^{1}, \alpha_{5}^{2}, \alpha_{5}^{3}, \alpha_{5}^{4}\right)=\left(\frac{1}{3}, \frac{1}{3}, \frac{1}{3}, \frac{1}{3}\right)$ and $\left(\alpha_{7}^{1}, \alpha_{7}^{2}, \alpha_{7}^{3}, \alpha_{7}^{4}\right)=\left(0, \frac{1}{3}, 0,0\right)$, i.e.,

$$
\begin{gathered}
a_{7}=\frac{1}{3} a_{2}+\beta_{7} b_{7}, \\
a_{8}=A\left(a_{7}\right)=\frac{1}{3} a_{2}+\beta_{7} A\left(b_{7}\right), \\
A\left(a_{8}\right)=\frac{1}{3} a_{2}+\beta_{7} A^{2}\left(b_{7}\right)=\frac{1}{3} a_{2}-\beta_{7} b_{7}-\beta_{7} A\left(b_{7}\right) \\
=a_{2}-a_{7}-a_{8} .
\end{gathered}
$$

This is of type 3.3. This completes the classification with $\mathbf{Z}_{3}$ holonomy. 
$\Psi=\mathbf{Z}_{4}$.

Choose $(A, a) \in \Gamma$ such that $A$ generates $\Psi, A(a)=a$, and $\|a\|$ minimal for this. As noted in the previous cases, $a \notin \Lambda$. We can diagonalize $\left.A\right|_{E^{\perp}}$ with $J$-eigenvalues $\pm i$. Hence $A^{2}+I: \mathbf{R}^{8} \rightarrow E$ and $\Lambda \cap E$ is a 4-dimensional lattice with generators $a_{1}$, $a_{2}, a_{3}, a_{4}$. Now $(A, a)^{4}=(I, 4 a)$ shows that $4 a \in \Lambda$, and by the minimality of $\|a\|$, we may choose these generators so that $a=a_{1} / 4$. To complete the set of lattice generators we use Lemma 3; the proof goes through in this case replacing $\pm \frac{\pi}{3}$ by $\pm \frac{\pi}{2}$.

Set $a_{j}=\alpha_{j}^{1} a_{1}+\alpha_{j}^{2} a_{2}+\alpha_{j}^{3} a_{3}+\alpha_{j}^{4} a_{4}+\beta_{j} b_{j}$ where $j=5,7, b_{j} \in E^{\perp}$, and the coefficients are real numbers. Since $A^{2} a_{j}+a_{j}=2 \alpha_{j}^{1} a_{1}+\cdots+2 \alpha_{j}^{4} a_{4} \in \Lambda$, we may reduce $\bmod \left\{a_{1}, a_{2}, a_{3}, a_{4}\right\}$ and see that the only possibilities for $\alpha_{j}^{i}$ are 0 or $\frac{1}{2}$ for $i=1,2,3,4$ and $j=5,7$. By an argument similar to the $\mathbf{Z}_{3}$ case, we can show that the only compact connected quaternionic Kaehler 8-manifolds with holonomy $\mathbf{Z}_{4}$ which can occur are of type 4.1, 4.2, or 4.3.

$\Psi=\mathbf{Z}_{6}$.

Choose $(A, a) \in \Gamma$ such that $A$ generates $\Psi, A(a)=a$, and $\|a\|$ minimal for this. As noted in the previous cases, $a \notin \Lambda$. We can diagonalize $\left.A\right|_{E^{\perp}}$ with $J$-eigenvalues $e^{( \pm \pi / 6) i}$. Hence $A^{2}-A+I: \mathbf{R}^{8} \rightarrow E$ and $\Lambda \cap E$ is a 4-dimensional lattice with generators $a_{1}, a_{2}, a_{3}, a_{4}$. Now $(A, a)^{6}=(I, 6 a)$ shows that $6 a \in \Lambda$, and by the minimality of $\|a\|$, we may choose these generators so that $a=a_{1} / 6$. To complete the set of lattice generators, we use Lemma 3 ; the proof goes through in this case replacing $\pm \frac{\pi}{3}$ by $\pm \frac{\pi}{6}$.

Set $a_{j}=\alpha_{j}^{1} a_{1}+\alpha_{j}^{2} a_{2}+\alpha_{j}^{3} a_{3}+\alpha_{j}^{4} a_{4}+\beta_{j} b_{j}$ where $j=5,7, b_{j} \in E^{\perp}$, and the coefficients are real numbers. Since $A^{2}\left(a_{j}\right)-A\left(a_{j}\right)+a_{j}=\alpha_{j}^{1} a_{1}+\cdots+\alpha_{j}^{4} a_{4} \in \Lambda$, we may reduce modulo $\left\{a_{1}, a_{2}, a_{3}, a_{4}\right\}$ and see that the only possibility for $\alpha_{j}^{i}$ is 0 , for all $i$ and $j$, i.e., $A\left(a_{6}\right)=a_{6}-a_{5}$ and $A\left(a_{8}\right)=a_{8}-a_{7}$. Hence $(A, a)$ and $\left(I, a_{k}\right)$, $k=2,3, \ldots, 8$,generate $\Gamma$ and the relations are given by type 6 .

5. Remarks. A. In higher dimensions, $\Gamma$ is more complicated. For example, $\Psi$ is not cyclic, in general, and is probably not even abelian. A positive statement we can make is that if $A \in \Psi$ and $\zeta_{n}$ (primitive $n$th root of unity) is a (J-) eigenvalue of $A$, then all primitive $n$th roots of unity are $(J-)$ eigenvalues of $A$. To see this, fix $J$ and diagonalize $A$ with $J$-eigenvalues $\{\zeta=\alpha+i \beta\}$. Since $A$ has finite order, $\zeta$ is a primitive (say, $n$ th) root of unity.

As a real matrix, $A$ consists of the $2 \times 2$ diagonal blocks

$$
\left[\begin{array}{cc}
\alpha & -\beta \\
\beta & \alpha
\end{array}\right]
$$

Hence the characteristic polynomial $\operatorname{det}(x I-A)$ is a product of the polynomials $x^{2}-2 \alpha x+1$. Note that $\zeta$ is a root of this quadratic. Since $\operatorname{det}(x I-A)$ has integer coefficients ( $A$ preserves a lattice), it follows that the $n$th cyclotomic polynomial (see $\S 3)$ divides $\operatorname{det}(x I-A)$.

B. It is fortunate that only 12 equivalence classes occur in dimension 8 . However it is no surprise that the number of classes is finite. According to Bieberbach [2], there is a finite number of affine diffeomorphism classes of the compact connected flat Riemannian $n$-manifolds (for any $n$ ). 
C. A complex manifold is projective if it can be complex analytically imbedded in a complex projective space. If $M$ is a quaternionic manifold then $M$ has a 2-sphere of complex structures $\left\{a J+b K+c J K \mid a^{2}+b^{2}+c^{2}=1\right\}$, and it is natural to ask which structures are projective. Sommese [9] has shown that at most countably many are projective.

D. A Hodge manifold $M$ is a Kaehler manifold whose Kaehler 2-form is in the image of the inclusion $H^{2}(M ; \mathbf{Z}) \hookrightarrow H^{2}(M ; \mathbf{R})$. The imbedding theorem of Kodaira [5] states that a compact Hodge manifold is projective. If $M$ is a quaternionic Kaehler manifold, then at most countably many of the complex structures are Hodge. In light of this, we shall say that a quaternionic Kaehler manifold is Hodge if at least one of the complex structures is Hodge.

It would be increasing to know if each of our 12 equivalence classes in Theorem 3 admits a Hodge representative. To see if a representative has a chance to be Hodge, first notice that if $\mathbf{R}^{8} / \Gamma$ is Hodge, then $\mathbf{R}^{8} / \Gamma^{*}$ is Hodge. Now one uses the Riemann conditions (see [3, p. 62]) to determine if the torus $\mathbf{R}^{8} / \Gamma^{*}$ is Hodge. The given representatives in Theorem 3 (i.e., the given representations of $\Gamma$ in $E(8)$ ) are not all Hodge.

\section{BIBLIOGRAPHY}

0. L. Auslander, Four dimensional compact locally Hermitian manifolds, Trans. Amer. Math. Soc. 84 (1957), 379-391.

1. L. Auslander and M. Kuranishi, On the holonomy group of locally Euclidean spaces, Ann. of Math. (2) 65 (1957), 411-415.

2. L. Bieberbach, Über die Bewegungsgruppen der Euklidischen Räume. I, Math. Ann. 70 (1911), 297-336; II, 72 (1912), 400-412.

3. S. S. Chern, Complex manifolds without potential theory, Van Nostrand, New York, 1967.

4. S. Kobayashi and K. Nomizu, Foundations of differential geometry, vols. I and II, Wiley, New York, 1963 and 1967.

5. K. Kodaira, On Kaehler varieties of restricted type, Ann. of Math. (2) 60 (1954), 28-48.

6. A. Kurosh, Lectures on general algebra, Chelsea, New York, 1965.

7. S. Lang, Algebra, 2nd ed., Addison-Wesley, Reading, Mass., 1967.

8. M. Obata, Affine connections on manifolds with almost complex, quaternionic or Hermitian structures, Japan. J. Math. 26 (1956), 43-79.

9. A. Sommese, Quaternionic manifolds, Math. Ann. 212 (1975), 191-214.

10. N. Steenrod, The topology of fibre bundles, 8th printing, Princeton Univ. Press, Princeton, N. J., 1972.

11. J. Wolf, Spaces of constant curvature, 2nd ed., McGraw-Hill, New York, 1972.

12. S.-T. Yau, Calabi's conjecture and some new results in algebraic geometry, Proc. Nat. Acad. Sci. U.S.A. 74 (1977), 1798-1799.

Department of Mathematics, Texas A \& M University, College Station, Texas 77843

Current address: Daniel H. Wagner, Associates, 1120 W. Mercury Boulevard, Hampton, Virginia 23666 